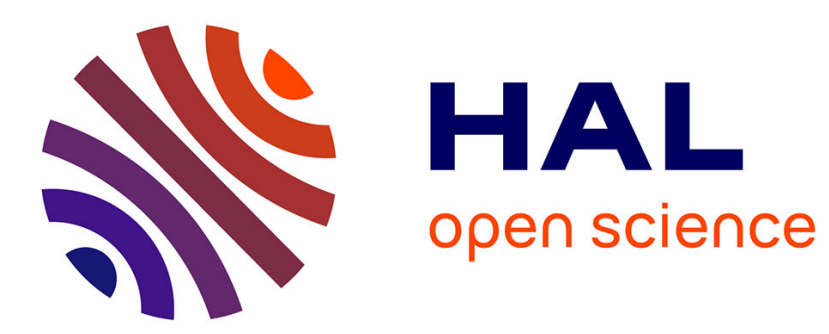

\title{
From the Characterization of Ranging Error to the Enhancement of Nodes Localization for Group of Wireless Body Area Networks
}

\author{
Anis Ouni, J Hamie, C Chaudet, Arturo Guizar, C Goursaud
}

\section{To cite this version:}

Anis Ouni, J Hamie, C Chaudet, Arturo Guizar, C Goursaud. From the Characterization of Ranging Error to the Enhancement of Nodes Localization for Group of Wireless Body Area Networks. EAI International Conference on Ad Hoc Networks (AdHocHets), Sep 2015, San Remo, Italy. pp.185-196, 10.1007/978-3-319-25067-0_15. hal-01246935

\section{HAL Id: hal-01246935 \\ https://hal.science/hal-01246935}

Submitted on 20 Dec 2015

HAL is a multi-disciplinary open access archive for the deposit and dissemination of scientific research documents, whether they are published or not. The documents may come from teaching and research institutions in France or abroad, or from public or private research centers.
L'archive ouverte pluridisciplinaire HAL, est destinée au dépôt et à la diffusion de documents scientifiques de niveau recherche, publiés ou non, émanant des établissements d'enseignement et de recherche français ou étrangers, des laboratoires publics ou privés. 


\title{
From the Characterization of Ranging Error to the Enhancement of Nodes Localization For Group of Wireless Body Area Networks
}

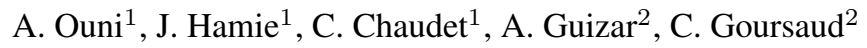 \\ 1 Institut Mines-Telecom, Telecom ParisTech, CNRS LTCI UMR 5141, 46 rue Barrault, 75634 \\ Paris, France, \\ anis.ounidtelecom-paristech.fr, \\ 2 University of Lyon, INRIA, INSA Lyon, CITI-INRIA, F-69621, Lyon, France
}

\begin{abstract}
Time-based localization in Wireless Body Area Networks (WBANs), has attracted growing research interest for the last past years. Nodes positions can be estimated based on peer-to-peer radio transactions between devices. Indeed, the accuracy of the localization process could be highly affected by different factors, such as the WBAN channels where the signal is propagating through, as well as the nodes mobility that bias the peer-to-peer range estimation, and thus, the final achieved localization accuracy. The goal of this paper consists in characterizing the impact of mobility and WBAN channel on the ranging and localization estimation, based on real mobility traces acquired through a motion capture system. More specifically, the ranging error is evaluated over all the WBANs links (i.e. on-body, off-body and body-to-body links), while an impulse Radio UltraWideband (IR-UWB) physical layer, as well as a TDMA-based Medium Access Control (MAC) are playing on. The simulation results show that the range measurement error can be modeled as a Gaussian distribution. To deal with the gaussianity observation of ranging error and to provide high positioning accuracy, an adjustable extended Kalman Filter (EKF) is proposed.
\end{abstract}

Key words: Body Area Networks, Group Navigation, Ultra Wideband, Ranging error, localization, EKF.

\section{Introduction}

The field of Wireless Body Area Networks has attracted much interest during the last years. Recently, these networks are considered for radio-location purposes thanks to their easy deployment. Unlike some costly and geographically restricted video acquisition system (e.g. Kinect, VICON [1]), WBANs are very suitable to work in noncontrolled indoor environments over large-scale body movements. In this localization context, several challenges are met, such as the need of high ranging and positioning accuracy. For those purposes, significant works have addressed the localization and navigation problem based on ranging estimation algorithms, while making direct and opportunistic use of the transmitted radio packets over Impulse Radio Ultra Wideband (IR-UWB) links or even Received Signal Strength Indicators (RSSI) over narrowbands links. The last solution is rather based on a prior model, which defines the variation 
of the received power as a function of the distance separating the involved devices. However, several studies show that the RSSI is not accurate enough for extracting the distances between devices [2]. On the opposite, IR-UWB benefits from high temporal and fine multipath resolution capabilities, which allow high precision on the estimation of the Time of Arrival (TOA) of the transmitted signal. Thus, the IR-UWB is promoted as a relevant physical layer for the localization applications [3]. Based on the TOA estimation, several ranging protocols lead for estimating the Round trip - Time of Flight (RT-TOF) based on $n$-way (i.e. 2-Way or 3-Way) transactions.

In this paper, we consider a group of mobile WBANs in navigation application occupied with a set of wireless devices. The positioning of the on-body nodes consists in collecting the range measurements either in a centralized manner (at a central node), or in a distributed scheme (each node collects its range measurements with respect to its neighborhood devices for its own localization). The range estimation through several transactions, as well as of the range collection from devices conducts for latency, which depends on the network size as well as the addressed scheduling [4]. In turn, this latency triggers ranging error, as the body can change its position and its gesture during this elapsed time. Besides, another main source of ranging error is related to the involved WBAN channel, in which the signal may suffer from NLOS propagation effects and dense multipath situation. These errors source, if not properly mitigated, generally yield severe degradation of positioning accuracy. The aim of this paper is twofold. Firstly, we characterize the impact of both mobility and WBAN channel on the ranging error with respect to on-, off- or inter-body links and, secondly, we propose an improvement of the final localization precision.

Due to the high difficulty of using radio-location experiments (i.e. lack of IEEE 802.15.6 integrated devices), most works of literature are focused on theoretical studies using often unrealistic assumptions and inaccurate abstraction of wireless communications, or focusing on a given layer, ignoring the other network layers. In this paper, we use a discrete-event simulator, WSNet [5], which takes into account the real time constraints for maintaining the peer-to-peer ranging transactions along the network and addresses all the layers. We implemented a complete protocol stack by crossing the physical up to the application layer dedicated to WBANs localization applications, particularly, based on IR-UWB physical layer with OOK modulation as defined by IEEE 802.15.6 and TDMA-based access control layer that mimics the scheduled access of a group of WBANs. We use a mobility model resulting from real experiments in which people movements were logged by a professional motion capture system. We considered a navigation group scenario composed of three WBANs. Our simulation results show that the measurement error range due to the nodes mobility can be modeled by a Gaussian distribution. To mitigate the effects of this error source and provide high positioning accuracy, an adjustable extended Kalman Filter (EKF) is proposed. Simulation results are included to show the capability of our improved EKF in increasing the positioning accuracy.

The rest of the paper is organized as follows. Next Section reviews related work. Section 3 describes the system model and gives the problem statement. In Section 4, we evaluate and characterize the ranging error obtained under realistic body mobility. 
Section 5, then, presents the core cooperative EKF, as well as our optimization approach and their localization performances. Finally, Section 6 concludes the paper.

\section{Related Work}

In the context of ranging estimation, [6] presents the issues of ranging error, position update latency and calculation algorithms under mobility. The authors are limited to the impact of MAC allocation resources on the capacity of the tracking system for Wireless Sensor Networks scenarios. In [7], the authors modeled the ranging error in terms of TOA estimation with real IR-UWB channel measurements in order to perform better localization algorithms but without any focus on the statistics of ranging error. [8] realizes a realistic measurement setup to achieve accurate positioning of WBAN nodes and compare the results with a Vicon system. The study, however, requires special attention on the ranging accuracy, i.e., characteristics of the on-body ranging error.

In the context of localization estimation, various positioning algorithms have been developed in the past few years. [9] has used the Non Linear Least Squares (NLLS) algorithm, which consists in minimizing a global quadratic cost function using the Gradient descent method incorporating the peer-to-peer range measurements. In [4], the problem of scheduling strategies at MAC layer is addressed to enable an Individual Motion Capture application with IR-UWB systems. The authors show that an effective scheduling scheme leads to estimate the nodes position one by one. The study, however, does not take into account the impact of channel noise on the position estimation. [10] and [11] adapt a centralized classical Multidimensional Scaling (MDS) for onbody motion capture applications and pose estimation. In [11], the authors introduce additional constraints relying on the prior knowledge of minimal and maximal feasible distances related to the body dimensions (and thus some kinds of geographical limitations). In [12] the centralized Maximum Likelihood estimator has been considered, introducing other constraints relying on the actual positions of on-body mobile nodes. More recently, the problem of cooperative localization based on the extended Kalman filtering $(\mathrm{EKF})$ has been developed which incorporates the cooperative peer-to-peer range measurements with the on-body nodes as well as the anchors [13]. The existing contributions do not exploit the potential information of the lower layers. In this paper, we investigate the robustness of the cooperative EKF in delivering high positioning accuracy for group navigation purposes while exploiting cross layer information, i.e., channel and nodes mobility.

\section{System model and Problem Formulation}

\subsection{System model and assumptions}

We consider a group of mobile WBANs and a set of fixed anchors (reference nodes) placed at known positions (position has been hard-coded into each anchor) with respect to a global 3D coordinate system. Each WBAN is defined by a set of on-body wireless devices, which are called on-body nodes. These on-body nodes are attached to 
the human body that evolves in an indoor and outdoor environment. The positions of all these on-body nodes are unknown and must be estimated relatively to the anchors. Since we assume a mesh topology network, we distinguish three kinds of links: either belonging to one single body network (on-body links), between distinct equipped users at reasonably short transmission ranges (inter-WBAN links), or even with respect to fixed elements of infrastructure (off-body links), as depicted in Figure 1. In this paper, all anchors and on-body nodes use an IR-UWB physical layer and operate with a single-channel frequency. In order to avoid the interference between communications, a TDMA-based MAC layer is used by all the nodes. We assume that the nodes positions are mapped into a stable Cartesian Local Coordinate System (LCS), which is defined by the fixed anchors and can be easily referenced to any Global Coordinate System (GCS). All the on-body nodes are then located in this coordinate system using peer-to-peer range measurements with the anchors or even between them, by performing TOA and 3-Way Ranging (3-WR) handshake protocol transactions. We refer to non-cooperative localization (resp. cooperative localization) when a node perform the 3-WR with the anchors only (resp. with the anchors and the other on-body nodes).

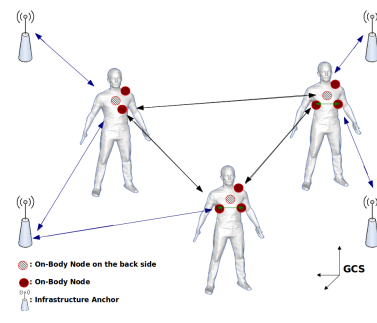

(a)

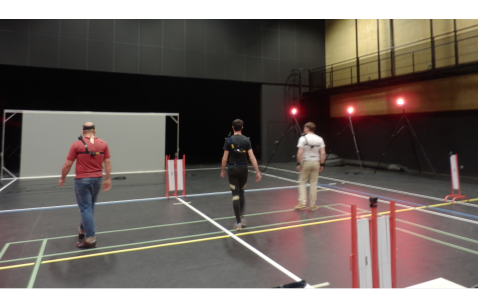

(b)

Fig. 1. Typical deployment scenario of group of WBANs in navigation application. There are three kinds of links: on-body links (green color), inter-body links (black color) and off-body links (blue color). The on-body nodes (red color) must be positioned relatively to the fixed anchors.

\subsection{Mobility Model derived from captured traces}

In this paper, we use a real mobility traces obtained from a measurement campaign conducted in the CORMORAN project. A Vicon optical motion capture system provides us the motion of people in a $13 \mathrm{mx} 8 \mathrm{~m}$ area. This system records all the markers positions thanks to 16 cameras surrounding the scene, and produces a C3D motion capture file containing all markers positions over time. Figure 1 illustrates this process, showing an image from the real scene with three persons in random navigation with the presence of four fixed anchors. Two subjects are equipped with four radios devices each, while the third subject is equipped with three devices (due to the limited number of devices). The devices are placed at the chest left, the chest right, the shoulder and the back. We equipped each wireless device with a marker in order to record its exact position alongside in addition to the movements of each subject. 


\subsection{Ranging Measurement and mobility problem}

The peer-to-peer range information is derived from RT-TOF estimation, which relies on 3-WR handshake protocol transactions and unitary TOA estimates for each involved packet [14]. The classical exchange for 3-WR is based on three guaranteed packets to evaluate the TOF between two nodes $i$ and $j$. Node $i$ starts by sending its request packet inside the assigned packet at time $\widetilde{T}_{i 0}$. Once this packet is received by node $j$ at time $\widetilde{T}_{j 0}$, node $j$ sends its first response back to the requester node $i$ inside its own dedicated packet at time $\widetilde{T}_{j 1}$, after a known delay. Node $i$ will receive this packet at time $\widetilde{T}_{i 1}$. To resolve the problem of clock drift, the responding node $j$ transmits a second response packet at time $\widetilde{T}_{j 2}$. This packet will be received by node $i$ at time $\widetilde{T}_{i 2}$. The resulting estimated TOF can be expressed as follows: $\widetilde{T O F}=\frac{1}{2}\left[\left(\widetilde{T}_{i 1}-\widetilde{T}_{i 0}\right)-\left(\widetilde{T}_{j 1}-\widetilde{T}_{j 0}\right)\right]-\frac{1}{2}\left[\left(\widetilde{T}_{i 2}-\widetilde{T}_{i 1}\right)-\left(\widetilde{T}_{j 2}-\widetilde{T}_{j 1}\right)\right]$.

The node $i$ can thus estimate its distance to node $j$ as : $\widetilde{d i j}_{i j}=\widetilde{T O F}_{i j} * c$, where $c$ denotes the speed of radio waves, i.e. $c=3 * 10^{8} \mathrm{~m} / \mathrm{s}$. Once all the distances separating the on-body nodes with the anchors and/or with the other on-body nodes are extracted, the on-body nodes positions can be estimated using a localization algorithm.

Given that these procedures have to be realized for each couple of devices, the traffic sent over the wireless medium quickly increases with the number of devices, using a classical peer-to-peer transaction handshake (P2P). To reduce the volume of control traffic, [15] has proposed a procedure called Aggregate-and-Broadcast (A\&B). They propose to mutualize control packets by letting each node initiates specific ranging transactions by broadcasting a request packet to all the other nodes, instead of querying each node separately. Each concerned nodes then aggregate its response and broadcasts a packet, which can play different roles (i.e. response 1, or even response 2). An intermediate solution, peer-to-peer ranging with request broadcasting (P2P-Broadcast), consists in broadcasting requests and transmitting responses one by one.

Another problem is the mobility of the nodes. In fact, as the on-body nodes are moving, it is straightforward that the distance between the nodes $i$ and $j$ can change between the beginning and the end of the 3 -WR (between $\widetilde{T}_{i 0}$ and $\widetilde{T}_{i 2}$ ), as well as the end of collecting all distances estimation. Therefore, the accuracy of the ranging estimation can be reduced. Besides, another main source of ranging error is related to the involved WBAN channel. Quantifying the ranging error due to the on-body nodes mobility is very beneficial for the phase of the resolution of the localization problem.

\subsection{WBAN Simulation Environment}

WSNET is a discrete-event simulator providing an advanced and complete simulation environment to evaluate networking protocols and wireless systems. Thanks to its modularity and its flexibility, WSNET offers the opportunity for developing and integrating our own modules and protocols, which could be in compliance with our WBAN context. At the radio layer stage, we implemented an IEEE 802.15.6 PHY UWB with OOK modulation and data rate of $0.4875 \mathrm{Mbps}$. In order to reduce the TDMA-frame duration, we evolved a dynamic slotted TDMA approach, where the duration of each slot depends 
on the size of the transmitted packet. To perform a ranging estimation, we added the 3WR protocol with the possibility of using the A\&B or P2P or P2P-B procedure. Finally, we used mobility traces acquired as explained in the subsection 3.2.

\section{Ranging Error Due to Mobility problem: Quantification and Distribution}

This Section aims to evaluate the impact of nodes mobility on the ranging estimation. The Root Mean Square Error (RMSE), computed as the absolute difference of measured and real distances, is quantified under realistic navigation scenarios. All simulation results are obtained over 20 independent trial runs. Over each run of 100s, the number of ranging updates between each pair of nodes is about 1300 times.

\subsection{Preliminary Study: Determining Elements of mobility}

To study the impact of the on-body nodes speed, we deployed 11 nodes in a single body as follows: one at the head, two at the torso, one at the back, one by hand, one by foot, one at the knee and one by elbow. Figures 2(a) illustrates the evolution of the cumulative distribution function (CDF) of the range RMSE of three different on-body links. As expected, the ranging error is more affected when the nodes mobility is considered as fast, confirming the importance of the mobility in the ranging action.

Figure 2(b) shows that the ranging accuracy also depends on the involved handshake transaction protocol. Indeed, we can observe an important gap between the performances of $\mathrm{A} \& \mathrm{~B}$ and $\mathrm{P} 2 \mathrm{P}$ or $\mathrm{P} 2 \mathrm{P}$ broadcast, while the performances between $\mathrm{P} 2 \mathrm{P}$ and $\mathrm{P} 2 \mathrm{P}-\mathrm{Broadcast}$ are closer. These results show that a higher ranging accuracy can be provided by grouping response packets rather than requests, which can be explained by the effect of reducing the required time for performing the $3 \mathrm{WR}$ over all nodes.

In the rest of this paper, we consider the group navigation scenario depicted in Figure 1 with A\&B protocol. Figure 2(c) illustrate the CDF of ranging error according to three kinds of links, on-, off- and inter-body links. It shows that the on-body ranging error is relatively negligible with respect to that of inter-body and off-body links. This observation refers to the fact that the nodes mobility is bounded at the body scale, but unbounded at the large scale where the body-to-body and the off-body links are involved. This observation is very important for cooperative context, since it leads to promote and enhance the use of on-body cooperation.

\subsection{Ranging Error Distribution due to nodes mobility}

The distribution of the ranging errors of the different kinds of links (on-, off- and interbody) can be approximated using an empirical statistics analysis. For this purpose, we firstly plotted the histogram and the empirical distribution of all ranging errors measured during 20 independent simulations of 100s each one. Figure 3 shows that the histogram and the empirical distribution closely follow a Normal distribution. The theoretical Normal distribution of each set of links is thus plotted with the mean value and 


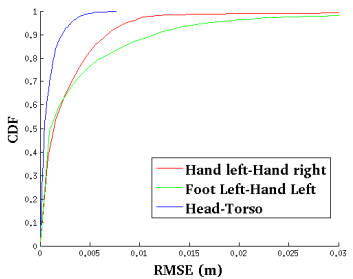

(a) The RMSE for different on-body links.

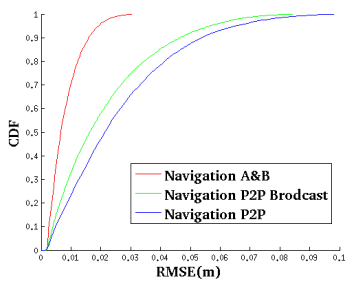

(b) The RMSE for different transaction protocols.

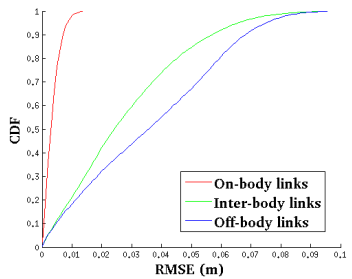

(c) The RMSE of on-, off- and inter-body links.

Fig. 2. CDFs of Root Mean Square Error (RMSE) of ranging errors. The impact of mobility on the ranging error depends on nodes speed, handshake transaction protocol and type of links.

the standard deviation of data. In accordance with the Figure 3 and table 4.2, the onbody links presents the smallest standard deviation, while the off-body links have the biggest. If we compare the empirical distribution and the theoretical distribution, we observe that the ranging measured errors due to the nodes mobility fits with a Normal distribution model with the specific parameters presented in table 4.2. These results are very important to improve the localization accuracy, and could be taken into account by the positioning algorithms. Moreover, normality distribution is usually assumed as a model for the ranging error in localization algorithms, like Extended Filter Kalman (EKF).

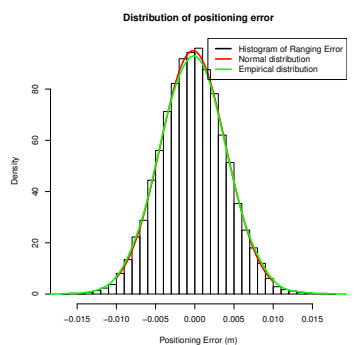

(a) On-body ranging error distribution

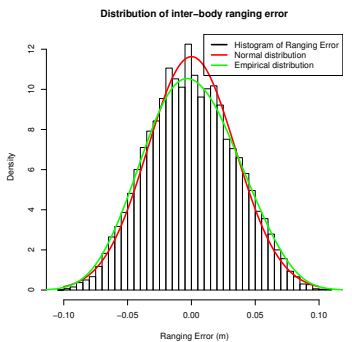

(b) Inter-body ranging error distribution

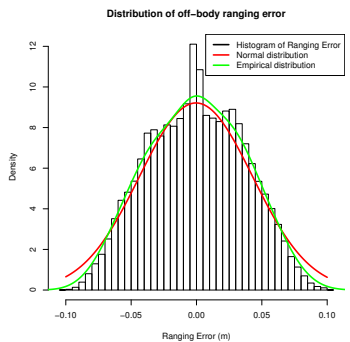

(c) Off-body ranging error distribution

Fig. 3. Distribution of ranging error for on-body, inter-body and off-body links

\begin{tabular}{|c|c|c|}
\hline On-body links & inter-body links & Off-body links \\
\hline \hline $\mathrm{N}(0,0.0042)$ & $\mathrm{N}(0,0.0343)$ & $\mathrm{N}(0,0.0433)$ \\
\hline
\end{tabular}

Table 1. Standard deviation of Ranging error due to nodes mobility 


\subsection{Impact of channel noise on Ranging Error}

WBAN Channel Abstraction In 3-WR, it is supposed that the receiver is able to detect accurately the pulse corresponding to the direct transmission between the nodes. However, depending on the person position, the channel might be multipath, inducing the use of the wrong path. However, modeling accurately the channel is computationally intensive. We therefore abstracted the channel by applying an error model directly to the range estimates. This error model, defined and characterized in [7] derives from UWB on-body and off-body channel measurements carried out in a pedestrian walking scenario. It supposes the use of an IEEE 802.15.6 mandatory band centered around $4 G H z$ with a bandwidth of $500 \mathrm{MHz}$. We assume that the ranging error is added to each distance at time stamp $t$, as follows:

$$
\widetilde{d_{i j}}(t)=d_{i j}(t)+n_{i j}(t)
$$

where $\widetilde{d_{i j}}(t)$ and $d_{i j}(t)$ are respectively the measured and the real distance between nodes $i$ and $j$ at time $t, n_{i j}(t)$ is a centered Gaussian random variable with a standard deviation $\sigma_{n}$.

\begin{tabular}{|c|c|c|}
\hline On-body links & inter-body links & Off-body links \\
\hline \hline $\mathrm{N}(0,0.1)$ & $\mathrm{N}(0,0.3)$ & $\mathrm{N}(0,0.3)$ \\
\hline
\end{tabular}

Table 2. Ranging error law due to the channel noise

Quantification of Ranging Error with/without channel noise We evaluate the average RMSE of ranging error in the case of ideal and noisy channel model for each set of links (on-, off- and inter-body). In the first case, Figure 4(a) shows a slow variation of the RMSE, especially, for the off- and inter-body links, while the on-body links presents a quasi-constant evolution with a mean value of $0.6 \mathrm{~cm}$ on RMSE. This slow variation of RMSE, can be explained by the limited variation of the on-body nodes mobility. We can also observe that the off-Body links are the most affected by the nodes mobility with a mean value of $3.6 \mathrm{~cm}$. Now by adding a noisy channel model (table 4.3), Figure 4(b) shows a fast variation of the average RMSE for each kind of links. We can observe that the impact of the channel on the ranging estimation is much higher than the impact of mobility (approximately multiplied by 10), thus the ranging error variation is dominated by the channel variation.

\section{Improvement of Extended Kalman Filter based on Ranging Error Due to Mobility}

In the last section, the ranging error due to nodes mobility is quantified and shown to follow a Gaussian distribution. To mitigate the effects of this error source and hence increase the positioning accuracy, we focus in this section to improve the performance of EKF algorithm by including the mobility standard deviations over all links. 


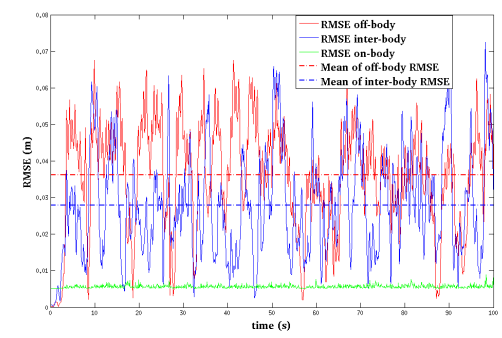

(a) RMSE ranging error with ideal channel.

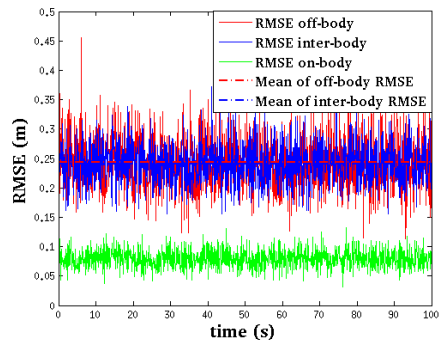

(b) RMSE ranging error with channel noise model

Fig. 4. Average RMSE of the all estimated distances for on-body, off-body and interinter-body links.

\subsection{Extended Kalman Filter Formulation with Full cooperative mode}

We assume that positioning measurements are realized every TDMA superframe duration, $T$, and we will denote by $\left\{X_{i}(k)\right\}_{i \in\{1 \ldots n\}}$ the set of the (unknown) 3D positions of the $n$ on-body nodes at time $t=k$.T. Let us also represent by $\left\{X_{i}\right\}_{i \in\{(n+1) \ldots(n+m)\}}$ the set of the 3D positions of the $m$ anchors. Now let us denote by $\widetilde{d}_{i j}(k)$ the value of the range measured at time $t=k . T$ between one on-body node $i \in\{1 \ldots n\}$ and another on-body node or anchor, $j \in\{1 \ldots m+n\}$. We regroup all the range measurements at step $k$ in a vector $\left.\left.\widetilde{\mathbf{d}}(k)=\left[\left\{\left\{\widetilde{d}_{i j}(k)\right\}_{j=n+1: n+m}\right\}_{i=1: n}\right\} \quad\left\{\left\{\widetilde{d}_{i j}(k)\right\}_{j=1: n}\right\}_{i=1: n}\right\}\right]$, which has a dynamic length depending on the number of available range measurements.

The TOA-based range measurements are defined as a non-linear functions of the on-body nodes' coordinates, thus we consider applying the well-know EKF solution [16], with the following state-space and observation models:

$$
\left\{\begin{array}{l}
\mathbf{S}(k)=\mathbf{A} \cdot \mathbf{S}(k-1)+\mathbf{u}(k) \\
\widetilde{\mathbf{d}}(k)=h(\mathbf{S}(k))+\mathbf{n}(k)
\end{array},\right.
$$

where $\mathbf{S}(k)=\left[\begin{array}{lllll}X_{1}^{T}(k) & V_{1}^{T}(k) & X_{2}^{T}(k) & V_{2}^{T}(k) \ldots X_{n}^{T}(k) & V_{n}^{T}(k)\end{array}\right]^{T}$ denotes the $6 n$ dimensional state-space vector at step $k$, that regroups the three-dimensional positions and velocities of nodes. $h($.$) is a function that materializes the non-linear relationship$ between the observed measurements and the state vector variables. The state transition matrix A, assuming locally linear movements in first approximation, is given by:

$$
\mathbf{A}=\mathbf{I}_{\mathbf{n}} \otimes\left(\mathbf{I}_{\mathbf{6}}+\left(\left(\begin{array}{ll}
0 & 1 \\
0 & 0
\end{array}\right) \otimes\left(\begin{array}{lll}
T & 0 & 0 \\
0 & T & 0 \\
0 & 0 & T
\end{array}\right)\right)\right)
$$

where $\mathbf{I}_{\mathbf{n}}$ denotes the $n$-dimensional identity matrix and $\otimes$ is the Kronecker product. Hence $\mathbf{A}$ accounts for some a priori information bridging the occupied positions at two consecutive steps $k$ and $k+1 . \mathbf{u}(k)$ is the state-space noise vector, whose covariance matrix is $\mathbf{Q} . \mathbf{n}(k)$ is the observation noise vector, whose covariance matrix $\boldsymbol{\Sigma}(k)$. 
Note that the latter can be adjusted dynamically over time depending on the availability and/or quality of the measurements. The implementation of this cooperative EKF follows a classical sequence of operations which involves the initialization, the prediction and the corrections phases as follows.

Prediction Phase:

$$
\left\{\begin{array}{l}
\hat{\mathbf{S}}(k \mid k-1)=\mathbf{A} \cdot \hat{\mathbf{S}}(k-1 \mid k-1) \\
\mathbf{M}(k \mid k-1)=\mathbf{A} \cdot \mathbf{M}(k-1 \mid k-1) \cdot \mathbf{A}^{T}+\mathbf{Q}
\end{array},\right.
$$

where $\hat{\mathbf{S}}(k \mid k-1)$ is the predicted state at step $k$ based on the latest available state estimate at step $k-1 \hat{\mathbf{S}}(k-1 \mid k-1)$, starting with the initial guess $\hat{\mathbf{S}}(0 \mid 0)$. $\mathbf{M}(k \mid k-1)$ is the corresponding prediction Minimum Mean Squared Error (MMSE) matrix.

Correction Phase:

$$
\mathbf{K}(k)=\mathbf{M}(k \mid k-1) \cdot \mathbf{H}^{T}(k) \cdot\left(\boldsymbol{\Sigma}(k)+\mathbf{H}(k) \cdot \mathbf{M}(k \mid k-1) \cdot \mathbf{H}^{T}(k)\right)^{-1},
$$

where $\mathbf{K}(k)$ is the filter gain and $\mathbf{H}(k)$ is the Jacobian observation matrix:

$$
\begin{gathered}
\mathbf{H}(k)=\left.\frac{\partial h(\mathbf{S}(k))}{\partial \mathbf{S}(k)}\right|_{\mathbf{S}(k)=\hat{\mathbf{S}}(k \mid k-1)}, \\
\left\{\begin{array}{l}
\hat{\mathbf{S}}(k \mid k)=\hat{\mathbf{S}}(k \mid k-1)+\mathbf{K}(k) \cdot(\widetilde{\mathbf{d}}(k)-h(\hat{\mathbf{S}}(k \mid k-1))), \\
\mathbf{M}(k \mid k)=\left(\mathbf{I}_{6 n}-\mathbf{K}(k) \cdot \mathbf{H}(k)\right) \cdot \mathbf{M}(k \mid k-1)
\end{array}\right.
\end{gathered}
$$

where $\hat{\mathbf{S}}(k \mid k)$ is the final state estimate at step $k$ based on the current prediction $\hat{\mathbf{S}}(k \mid k-$ 1) and observation $\widetilde{\mathbf{d}}(k)$, and $\mathbf{M}(k \mid k)$ is the related estimation MMSE matrix.

\subsection{Adjusted EKF}

The last EKF version, takes only into account the range measurement error generated by the channel. However, as the nodes mobility affects the ranging estimation and hence the positioning accuracy, we propose to compensate this ranging errors by adjusting the marginal diagonal elements of the measurement noise covariance matrix $\Sigma(k)=$ $\left[\hat{\sigma}_{i i}(k)\right]$. More specifically, our proposal consists in adjusting the covariance matrix, while taking into account the mobility effects over each of on-, off- and body-to-body links. Thus, the mobility standard deviations over all links (i.e. $\hat{\sigma}_{\text {on }}, \hat{\sigma}_{\text {off }}$ and $\hat{\sigma}_{\text {int }}$ ) are incorporated by the system after the correction phase as follows:

$$
\hat{\sigma}_{i i}(k)=\left\{\begin{array}{lc}
\left(\hat{\sigma}_{c h}(k)+\hat{\sigma}_{o n}\right)^{2}, & \text { on - body links } \\
\left(\hat{\sigma}_{c h}(k)+\hat{\sigma}_{i n t}\right)^{2}, & \text { inter - body links }, \\
\left(\hat{\sigma}_{c h}(k)+\hat{\sigma}_{o f f}\right)^{2}, & \text { off }- \text { body links }
\end{array}\right.
$$

where $\sigma_{c h}$ is the standard deviation of the ranging error due to the channel component. 


\subsection{On-body node's estimation with full-Cooperative EKF}

In Section 4, we have shown that the ranging errors follow a Gaussian distribution, where each kind of WBAN links (on-, off-, and inter-body links) is characterized by its proper standard deviation. In this subsection, we evaluate the resulting gain from the improvement of Kalman filter, while involving the effects of mobility over all links. Using a full cooperative scenario (on-, off- and inter-body links), Figure 5 shows the CDF of the RMSE resulting from the use of the conventional and the adjusted (improved) EKF. This last figure shows that our proposal permits to improve the localization accuracy, where the average goes from $0.487 \mathrm{~m}$ to $0.442 \mathrm{~m}$ (improvement with a rate of $9.1 \%$ ). The positive effect of our proposal is also seen by averaging the resulting RMSE of nodes attached to each body. This fact is represented in Figure 6, where the blue bars (resp. red bars) represents the average nodes RMSE over each body, while using the conventional EKF (adjusted EKF). As shown, our proposal improves the localization performances over all the incorporated body in our group of mobile WBAN with a gain of, respectively, $9.88 \%, 9.28 \%$ and $11.22 \%$.

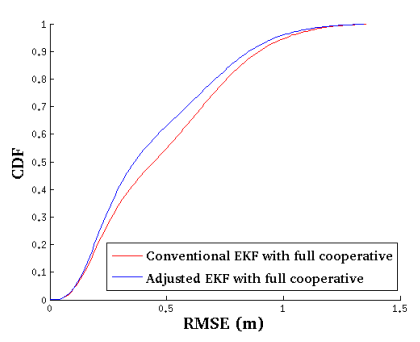

Fig. 5. CDFs of estimated on-body node's RMSE for full-cooperative multi-user navigation scenario

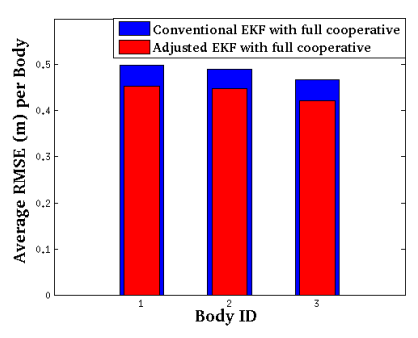

Fig. 6. Average RMSE of the estimated nodes positions per body with full cooperative EKF with and without ranging error mitigation

\section{Conclusion}

This paper focused on nodes localization problem for group of WBANs, based on peerto-peer range measurements fed through impulse Radio-Ultra Wideband and time of arrival estimation. We firstly quantified and characterized the error of on-, off- and interbody distances estimations, based on real mobility model. Based on extensive measurement, our results showed that the ranging errors can be modeled as a Gaussian distribution. To deal with the gaussianity observation of ranging error and to provide high positioning accuracy, we secondly proposed an adjustable cooperative EKF algorithm. Our simulation results showed an improvement between $9 \%$ and $11 \%$ of localization accuracy. 


\section{Acknowledgment}

This work has been carried out in the frame of the CORMORAN project, which is funded by the French National Research Agency (ANR) under the contract number ANR-11-INFR-010.

\section{References}

1. "Vicon." http://www.vicon.com/.

2. "Ieee 802.15 wpan task group 6 (tg6) - body area networks."

3. S. Gezici and Al., "Localization via ultra-wideband radios: a look at positioning aspects for future sensor networks," Signal Processing Magazine, vol. 22, no. 4, pp. 70-84, July 2005.

4. A. Guizar, A. Ouni, C. Goursaud, N. Amiot, and J. Gorce, "Impact of MAC scheduling on positioning accuracy for motion capture with UWB body area networks," Proceedings of the 9th International Conference on Body Area Networks, 2014.

5. G. Chelius, A. Fraboulet, and E. Ben Hamida, "http://wsnet.gforge.inria.fr/."

6. J. Choliz, A. Hernandez, and A. Valdovinos, "A framework for UWB-based communication and location tracking systems for wireless sensor networks," Sensors, vol. 11, pp. 90459068, Sep 2011.

7. J. Hamie, B. Denis, R. D'Errico, and C. Richard, "On-body TOA-based ranging error model for motion capture applications within wearable UWB networks," Journal of Ambient Intelligence and Humanized Computing, Dec. 2013.

8. C. G. P. J. B. R. Bharadwaj, S. Swaisaenyakorn and A. Alomainy, "Localization of wearable ultrawideband antennas for motion capture applications," Antennas Wirel. Propag. Lett., vol. 13, pp. 507-510, 2014.

9. E. Ben Hamida, M. Maman, B. Denis, and L. Ouvry, "Localization performance in Wireless Body Sensor Networks with beacon enabled MAC and space-time dependent channel model," in IEEE 21 st International Symposium on Personal, Indoor and Mobile Radio Communications (PIMRC) Workshops, (Istanbul, Turkey), Sept. 2010.

10. H. A. Shaban, M. A. El-Nasr, and R. M. Buehrer, "Toward a Highly Accurate Ambulatory System for Clinical Gait Analysis via UWB Radios," IEEE Transactions on Information Technology in Biomedicine, vol. 14, Mar. 2010.

11. M. Mhedhbi, M. Laaraiedh, and B. Uguen, "Constrained LMDS technique for human motion and gesture estimation," in 9th Workshop on Positioning Navigation and Communication (WPNC), (Dresden, Germany), Mar. 2012.

12. Z. W. Mekonnen, E. Slottke, H. Luecken, C. Steiner, and A. Wittneben, "Constrained maximum likelihood positioning for uwb based human motion tracking," in International Conference on Indoor Positioning and Indoor Navigation (IPIN), Sept 2010.

13. J. Hamie, C. Chaudet, and B. Denis, "Improved Navigation Capabilities in Groups of Cooperative Wireless Body Area Networks," in 9th International Conference on Body Area Networks (BodyNets), (Oslo, Norway), Sept. 2014.

14. M. Maman, B. Denis, M. Pezzin, B. Piaget, and L. Ouvry, "Synergetic MAC and higher layers functionalities for UWB LDR-LT wireless networks," in IEEE International Conference on Ultra-Wideband (ICUWB 2008), vol. 3, pp. 101-104, 2008.

15. D. Macagnano, G. Destino, F. Esposito, and G. Abreu, "MAC performances for localization and tracking in wireless sensor networks," 2007 4th Workshop on Positioning, Navigation and Communication.

16. T. Perälä and R. Piché, "Robust extended Kalman Filtering in Hybrid Positioning Applications," in 4th Workshop on Positioning, Navigation and Communication (WPNC'07), (Hannover, Germany), Mar. 2007. 